considerably improve on the speed and therefore the feasibility of trial and error testing of real problems-an approach that is still necessary when geophysical results can often be interpreted by an infinity of possible models of which the most geologically sensible must then be sought. So far such an evaluation is still a human perogative, although one wonders for how much longer! Mussett and Dagley (Liverpool) showed how the volcanic activity of Mull is now confined to within 3 million years some 60 million years ago. Banks and Swain (Lancaster \& Leicester) raised the problem of the source of isostatic compensation, a problem of major importance since the inception of plate tectonics and therefore the potentially great depths of the levels of isostatic compensation on the continents. Studies of the gravity field in East Africa now suggest that while the depth of compensation for the plateau is unknown, the smaller structure of the Kenya dome, on which the rift valleys lie, is compensated at fairly shallow depths, less than $55 \mathrm{~km}$ - such results being consistent with results from North America which indicate that gravity anomalies of long wavelengths, $\sim 1,000 \mathrm{~km}$ or more, have perfect isostatic compensation, but wavelengths of less than $100 \mathrm{~km}$ are only imperfectly compensated. Rice (Newcastle) questioned the standard gravitational segregation model for the Skaergaard intrusive and provided a convective alternative that appeared equally adept at explaining volcanic differentiation or homogenising salad oil.

\section{Turning points in predicted protein structures}

\section{from $M$. Geisow}

IN recent years, considerable improvements have been made in the accuracy of protein conformation derived from amino acid sequence data alone. The widespread adoption of this approach may be judged by the number of recent reviews, including two from authors in laboratories specialising in the experimental determination of protein 3-D structure (Shulze Angew. Chem. 16, 23; 1977, Sternberg \& Thornton Nature 271, 15; 1978).

In the most general terms, there are two empirical approaches to the estimation of protein folding from sequence information. The best known of these uses the frequencies with which particular amino acids occur in $\alpha$-helix, $\beta$-structure or $\beta$-turns (180 ${ }^{\circ}$ reversals of the polypeptide chain) in proteins of known conformation. B. Robson at the University of Manchester and $P$. Chou and G. Fasman at Brandeis University use these statistics to determine the likely location of the same structural features in proteins of unknown 3-D structure. The other broad approach is intrinsically kinetic and is founded upon the assumption that when proteins begin to fold, local interactions (between nearby amino acids) predominate over interactions between amino acids more distantly separated in linear sequence. Many workers have used this assumption as the basis for a predictive method and V. Lim, of the USSR Academy of Sciences, has published a set of rules which allow the estimation of the total folding of globular proteins.

Since none of these methods yet competes with crystallography in precision of structure determination, their value to biochemists is restricted to applications which only require approximate structural information. A good example of the use of the empirical approach is the independent estimation of protein secondary structure for comparison with that determined by spectroscopic methods. Regions of sequence which lack regular structure can also be estimated and these indicate likely sites for proteolytic attack.

This type of strategy has been adopted by Beely (Biochem biophys. Res. Commun. 76, 1051; 1977) and Aubert et al. (Arch. Biochem. Biophys. 175,$410 ; 1976$ ) who find that sugar chains seem to be preferentially attached to glycoproteins at $\beta$-turns. Beely reports that 30 out of 31 glycosylated residues occur in sequences favouring the $\beta$-turn. He points out that although the amino acid sequence Asn (sugar)-X-Ser (or Thr) is a necessary condition for glycosylation, it is not sufficient, and his analysis points to an additional conformational requirement. Carbohydrate is present at $\beta$-turns in the known structures of human immunoglobulin heavy chain and RNase B. Small et al. (Biochem. biophys. Res. Commun. 79, 341; 1977) find that $80 \%$ of amino acids phosphorylated by protein kinases are also located in sequences predicted to be in the $\beta$-turn conformation.

It may be that the enzymes responsible for these post-translational modifications require a particular conformation of their target amino acids, but an overriding consideration must be that such sequences are exposed at the surfaces of their protein substrates. The $\beta$-turn is certainly a surface struc-

M. Geisow is at the National Institute for Medical Research, London. ture, but it is only a special example of the often irregular bends and loops which comprise a substantial fraction of the surface of globular proteins. Although these features are poorly located by available treatments they are just the regions of chain most likely to be involved in intermolecular events.

An improvement in the location of these bends has been introduced recently by Rose (Nature 272, 586; 1978). When the free energies of transferring each amino acid side chain from an aqueous to a non-polar environment are plotted as a function of linear sequence, turns and loops appear as local minima, between maxima which represent regions of the polypeptide chain which form the hydrophobic protein core. This is a welcome adjunct to the current empirical prediction techniques, since the algorithm described has a firm thermodynamic basis.

With the help of these methods many potential sites of post-translational modification, such as limited proteolysis or adenylylation, can be explored using existing amino acid sequence information. However, the correlation of acceptor sequences with predicted structural features should be made with some caution. One must first be satisfied that the modification of interest occurs after the full tertiary structures of the molecules are acquired. For example, Linguppa et al. (Proc. natn. Acad. Sci. U.S.A. 75, $2338 ; 1978)$ show that the glycosylation of $\alpha$-lactalbumin only occurs during its synthesis on the ribosome. Pless et al. (Proc. natn. Acad. Sci. U.S.A. 74, 134; 1977) find that a variety of glycoproteins can only serve as carbohydrate acceptors if they are supplied to the enzyme system in their denatured state. The lack of structure during this particular modification obviously weakens the argument for a structural basis of the observed specificity. With this proviso, the methods discussed here may well prove powerful in locating or confirming potential sites of biochemical modification of globular proteins.

\section{0 years ago}

IT is stated in the French papers that Mr. Edison is to have no reward whatever at the Paris Exhibition for his phonograph. The reasons alleged for this apparent denial of justice are somewhat amusing. The jury of the class of instruments of precision declared that the phonograph could not be considered as at all an instrument of precision, but merely a toy; consequently they sent it to the class of telegraphy to be rewarded. But the telegraphists replied that it was of no use whatever in telegraphy, and refused to examine it. The consequence is that the most wonderful invention, probably, in the Exhibition, will be passed by unmentioned and unrewarded.

From Nature 18, 15 August, 424; 1878. 